\title{
Evaluation of functional results according to Body Mass Index in patients with acute non-specific low back pain
}

\author{
Yücel Yildirim ${ }^{\mathrm{a}, *}$, Bilge Kara ${ }^{\mathrm{a}}$, Arzu Genç $^{\mathrm{a}}$, Zeliha Başkurt ${ }^{\mathrm{a}}$, Ferdi Başkurt ${ }^{\mathrm{a}}$ and Serhat Erbayraktar ${ }^{\mathrm{b}}$ \\ ${ }^{\mathrm{a}}$ Dokuz Eylül University, School of Physical Therapy, Izmir, Turkey \\ ${ }^{\mathrm{b}}$ Dokuz Eylül University, School of Medicine, Neurosurgical Department, Izmir, Turkey
}

\begin{abstract}
Objective: The aim of our study was to detect the factors which might affect the recovery of the patients with Acute Non-Specific Low Back Pain [ANSLBP] according to their Body Mass Index [BMI], and to determine the effects of these factors on disability and the perceived functional level with quality of life.

Design: A retrospective study.

Patients: Ninety-three patients were included in the study, and separated as three groups according to BMI [normal weight group, Grade1 obesity group, Grade 2 obesity group].

Methods: Acute Low Back Pain Screening Questionnaire [ALBPSQ] and SF-36 Physical Functioning Scale [PFS] were used in the evaluation of the patients.

Results: The ALBPSQ and SF-36 PFS results of our patients in all three groups before and after the treatment were found to be significant in the direction of positive scores $[p<0.05]$. When the SF-36 PFS scores and the total scores of ALBPSQ were compared among the groups according to the increase of the obesity, there existed a significant difference $[p<0.05]$. Between the ALBPSQ and SF-36 PFS scores, in the three groups correlation was determined in the negative direction.

Conclusion: With the treatment applied to our patients, pain and disability with the quality of life showed positive differences, but the results were observed to be affected unfavorably through the increase of obesity based on the functional status and decrease in the quality of life.
\end{abstract}

Keywords: Acute low back pain, function, quality of life

\section{Introduction}

Obesity is one of the several lifestyle factors that have been suspected of causing low back pain [LBP]. In many epidemiologic studies, investigators have tested the association between obesity and Non-specific LBP [NLBP], but the results vary among studies [1]. However, a look at the literature reveals some confusion. There are several hypotheses relating to a link between obesity and LBP. Increased mechanical de-

*Address for correspondence: Yücel Yildirim, Assistant Professor, PT, Dokuz Eylül University, School of Physical Therapy 35340, İnciraltı, İzmir, Turkey. Tel.: +90 23241249 31; Fax: +90 232270 50 30; E-mail: yucel.yildirim@deu.edu.tr. mands resulting from obesity have been suspected of causing LBP through excessive wear and tear, and it has been suggested that metabolic factors associated with obesity may be detrimental [2-5].

Specifically, low back pain has been correlated with obesity in many studies. Although the association between low back pain and body weight has been well studied, little is known how obesity affects functional status in spine patients $[9,11,14,17]$. Standard clinical parameters are used to examine patients across all levels of BMI in an effort to understand how obesity is related to the health status and functional level of spine patients.

Our study was designed to obtained answers to the following questions: 
- Is there any relationship between the functional status and quality of life of the patients and obesity?

- Is the activity of the practiced physiotherapy program varied according to the obesity level?

\section{Methods}

\subsection{Patients}

Upon studying the files of the outpatients admitted to the physical therapy clinic of the university hospital in November 2004 through January 2005, those who had pain more than 12 weeks, those who had operation about lumbar region, and those having trauma, tumors, infection and inflammatory diseases, and finally those with a special condition such as pregnancy were excluded from the evaluation. The results of the remaining 93 outpatients were taken under evaluation.

\subsection{The grouping of the patients}

The World Health Organization international classification of obesity defines the levels of severity as follows: normal range [BMI $<25.0 \mathrm{~kg} / \mathrm{m}^{2}$ ], Grade 1 [moderate obesity $25.0-29.9 \mathrm{~kg} / \mathrm{m}^{2}$ ], Grade 2 [severe obesity $30.0-39.9 \mathrm{~kg} / \mathrm{m}^{2}$ ], and Grade 3 [morbid obesity $\left.>40.0 \mathrm{~kg} / \mathrm{m}^{2}\right]$.

The following formula was used in calculating;

$\mathrm{BMI}=$ body weight/height $\mathrm{x}$ height

According to the above formula, our patients were taken under study in three groups:

Group 1: normal weight; Group 2: Grade 1 obesity; Group 3: Grade 2 obesity [6,11].

\subsection{Measurements}

To all the patients applying to our clinic, the assessments of Acute Low Back Pain Screening Questionnaire [ALBPSQ] and SF-36 Physical Functioning Scale [SF-36 PFS] are routinely made before and after the treatment.

\subsection{Acute Low Back Pain Screening Questionnaire [ALBPSQ]}

The ALBPSQ, a new self-administered measure of biopsychosocial factors related to LBP chronicity, was used to establish each patient's degree of biopsychosocial risk for poor outcome before commencement of therapy. This questionnaire is composed of 24 items divided into five groups [function, pain, psychological factors, fear avoidance, and miscellaneous]. Twentyone of the 24 items are scored on a 0 [good] to 10 [bad] scale, yielding a total score range of 0 to $210[13,15]$. The high scores showed the bad result in ALBPSQ.

\subsection{SF-36 Physical Functioning Scale [SF-36 PFS]}

A back specific SF-36 Physical Functioning scale would combine the advantages of both generic and specific functional questionnaires into a single, parsimonious set of items from which the original 10-item scale could still be extracted. The possible total score range from 0 to 100, with a higher score indicating better function. For the SF-36 PFS, items were scored so that 'yes', limited a lot' scored 0, 'yes, limited a little' scored 1, and 'no, not limited at all' scored 2 in section 1 . Sum the score in section 1 only divided by 20 , multiply by 100 . Sum the individual item scores divide by 60 multiply 100 [7].

\subsubsection{Physiotherapy management}

Patients received treatment as determined by their therapist. Physiotherapy management of ANLBP incorporates a broad range of therapeutic strategies [e.g., advice, education, exercise therapy, electrotherapy, and heat therapy]. The physiotherapy program was practiced every day during three weeks $[13,18]$.

\subsection{Statistical analysis}

Descriptive statistics include frequency distribution for categorical variables and means, medians ranges and standard deviations [SDs] for continuous variables. Wilcoxon Signed Rank test was used in the comparison of ALBPSQ and SF-36 PFS results in each group before and after the therapy. The correlation between the ALBPSQ and SF-36 PFS was detected by Pearson Correlation Analysis in each three groups. The amounts of increase in the groups according to the before treatment were compared by using Mann-Whitney U test and ANOVA. In all cases, $p<0.05$ was considered to be significant. The statistical analysis was performed using the statistical package for social sciences [SPSS] version 11.0. 
Table 1

The demographic characteristics of groups

\begin{tabular}{lcccc}
\hline & Groups & Normal Weight & Grade 1 Obesity & Grade 2 Obesity \\
\hline Age [years] & Mean \pm SD & $49.40 \pm 14.50$ & $49.40 \pm 14.50$ & $52.52 \pm 9.17$ \\
Gender $[n]$ & Female - Male & $18-12$ & $18-12$ & $30-14$ \\
Work Status $[n]$ & $a-b-c$ & $8-10-12$ & $8-10-12$ & $17-13-14$ \\
\hline
\end{tabular}

Work Status: a; House wife, b; Retired, c; Actively Working.

Table 2

Values Presented as Means, SD and p of SF -36 Scores of Groups

\begin{tabular}{lccc}
\hline SF-36 PFS $[0-100]$ & Before Treatment & After Treatment & p \\
\hline Normal Weight Group & $56.46 \pm 18.60$ & $72.80 \pm 15.70$ & $S$ \\
Grade 1 Obesity Group & $58.05 \pm 19.71$ & $71.39 \pm 16.83$ & $S$ \\
Grade 2 Obesity Group & $45.19 \pm 14.51$ & $61.83 \pm 17.12$ & $S$ \\
\hline
\end{tabular}

$\mathrm{S}: p<0.05$ significant.

Table 3

Values Presented as Means, SD and p of ALBPSQ Scores of Groups

\begin{tabular}{lcccc}
\hline \multicolumn{1}{c}{ ALBPSQ [0-210] } & & Normal Weight Group & Grade 1 Obesity Group & Grade 2 Obesity Group \\
\hline Pain & BT & $32.23 \pm 7.29$ & $30.97 \pm 6.97$ & $33.57 \pm 7.38$ \\
& AT & $28.76 \pm 5.26$ & $26.38 \pm 6.18$ & $27.52 \pm 6.66$ \\
Psychological Factors & BT & $12.73 \pm 5.57$ & $13.43 \pm 5.37$ & $14.26 \pm 4.77$ \\
& AT & $10.23 \pm 5.52$ & $10.70 \pm 4.31$ & $11.36 \pm 4.07$ \\
Fear Avoidance & BT & $15.46 \pm 8.06$ & $16.38 \pm 7.14^{*}$ & $18.10 \pm 7.01^{*}$ \\
Function & AT & $15.93 \pm 7.27^{*}$ & $14.68 \pm 6.99^{*}$ & $17.63 \pm 5.19^{*}$ \\
Miscellaneous & BT & $20.30 \pm 14.03$ & $16.59 \pm 15.61$ & $21.73 \pm 14.41$ \\
& AT & $11.70 \pm 9.35$ & $10.84 \pm 10.64$ & $16.00 \pm 9.75$ \\
Total Score & BT & $27.10 \pm 6.83$ & $24.88 \pm 7.63$ & $28.84 \pm 6.61$ \\
& AT & $23.10 \pm 7.07$ & $20.70 \pm 7.49$ & $24.47 \pm 8.11$ \\
& BT & $108.53 \pm 26.62$ & $102.02 \pm 28.93$ & $114.78 \pm 28.28$ \\
\hline
\end{tabular}

${ }^{*} p>0.05$ non-significant. BT: Before Treatment and AT: After Treatment.

\section{Results}

The patients' demographic characteristics such as age, gender, work status were recorded [Table 1].

The ALBPSQ and SF-36 PFS results before and after treatment of our patients whom we discriminate according to their BMI as normal group, Grade 1 obesity group, and Grade 2 obesity group were found to be significant in all three groups in the direction of favorable scores $[p<0.05]$.

When the before treatment SF-36 PFS scores were examined, the best result was observed in Grade 1 obesity group $(58.05 \pm 19.71)$, the worst score was obtained in Grade 2 obesity group. In the examination of after treatment scores; the best score was seen in normal weight group $(72.80 \pm 15.70)$, Grade 1 obesity and Grade 2 obesity group respectively (Table 2 ). When the before treatment and after treatment scores were compared of the groups significant differences were found $[p<0.05]$.

When the before treatment scores of ALBPSQ were examined; the worst result was observed in Grade 2 obesity group $(114.78 \pm 28.28)$, the best score was seen in Grade 1 obesity group $(102.02 \pm 28.93)$. And in the examination of the after treatment scores; the best score was seen in Grade 1 obesity group $(83.56 \pm 24.53)$; normal weight group $(89.66 \pm 20.64)$ and Grade 2 obesity group $(100.73 \pm 25.51)$ respectively (Table 3$)$ (Fig. 1). When all the sub-scores of ALBPSQ were examined; there were significant differences $[p<0.05]$ except fear avoidance parameter $[p>0.05]$.

The ALBPSQ and SF-36 PFS scores of our patients showed correlation negatively at $p<0.01$ level.

\section{Discussion}

In the literature studies, while BMI, known as the rate of body weight to the height, is pointed out as the risk factor, in a few studies its link with LBP has been stressed [14]. However, obese patients have significantly lower functional status than no obese patients; that is, the obese have more physical morbidity than their normal weight counterparts $[10,17]$. Our study 


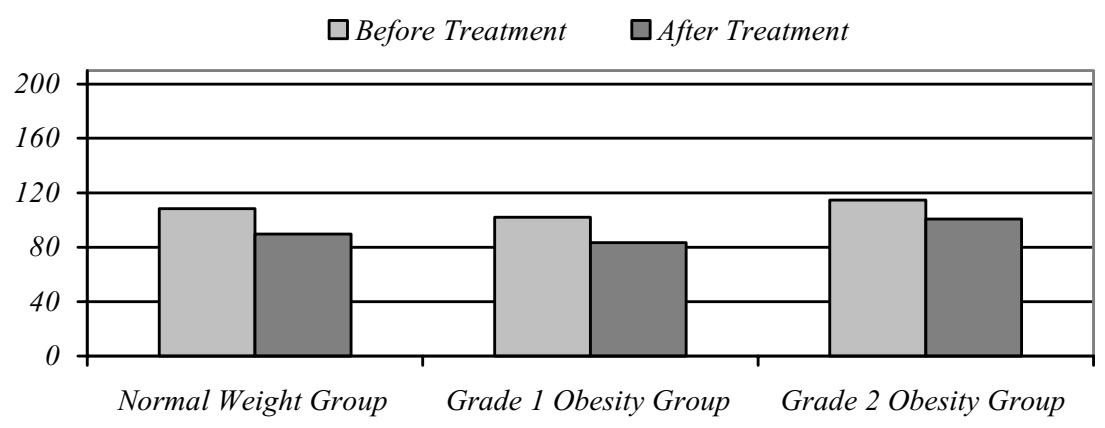

Fig. 1. Comparison of Total Scores of ALBPSQ in Groups at Before and After Treatment.

was planned for the purpose of examining the differences in the responses to the treatment, and in their obesity in parallel with their functionalities of our patients who were grouped according to their BMIs.

When the average of ages and working status of our patients are examined, it was observed that along with getting older an increase in their obesity developed, and inactivity in their work status started. In parallel with this, as the obesity rate of the patients increased, it was observed that the total scores of SF-36 PFS and ALBPSQ were negatively affected. This correlation between the quality of life and functional status showed us that the negative changes in the working life and functional status observed along with aging caused parallel decreases in the quality of life [8].

When it was studied how our patients' quality of life and functionalities changed through our treatment we practiced, in all the assessments of the groups positive increases were observed compared with the treatment practiced beforehand. These increases observed before treatment weren't influenced by the obesity degree of our patients. When the negative effects of pain was removed by our treatment practiced on the patients, it was observed that in the quality of life and functionality levels of our patients, there were some increases compared to the time before treatment. Because of the functionality which is affected negatively with the obesity degree, these type of patients showed be directed toward more active type of life along with the physiotherapy.

It might seem obvious that both pain and disability determine quality of life in patients with LBP. However, other factors unrelated to LBP may influence quality of life, there is sparse evidence on the correlation between LBP and decrease in quality of life, and there are few data on the correlation between pain and disability with quality of life $[12,16]$. There is sparse evidence on the correlation between LBP and decrease in quality of life, there is sparse evidence on the correlation between
LBP and decrease in quality of life, and also few data exist on the correlation between pain and disability with quality of life.

The results of our study indicated that through the treatment practiced on our patients, positive advances were observed on disability together with pain and the quality of life, but it was found out that with the increase of obesity, in connection with the decrease of functional status and the quality of life, the results were negatively affected. In order for the treatment activity to be longer in time and permanent, we still need more study in the direction of removing the negative impacts of obesity firstly by suggesting changing the living patterns of our patients aiming to decrease their quality of lives.

\section{References}

[1] T. Adera, R.A. Deyo and R.J. Donatella, Premature menopause and low back pain. A population- based study, Ann Epidemiol 4 (1994), 416-422.

[2] S. Aro and P. Leino, Overweight and musculoskeletal morbidity: A ten-year follow-up, Int J Obes 9 (1985), 267-275.

[3] H. Bergenudd, B. Nilsson, A. Udén and S. Willner, Bone mineral content, gender, body posture, and build in relation to back pain in middle age, Spine 14 (1989), 577-579.

[4] F. Biering-Sorensen, Physical measurements as risk indicators for low-back trouble over a one-year period, Spine 9 (1984), 106-119.

[5] S.J. Bigos, D.M. Spengler, N.A. Martin, J. Zeh, L. Fisher and A. Nachemson, Back injuries in industry: A retrospective study. III. Employee-related factors, Spine 11 (1986), 252256.

[6] O.M. Bostman, Body mass index and height in patients requiring surgery for lumbar intervertebral disc herniation, Spine $\mathbf{1 8}$ (1993), 851-854.

[7] M. Davidson, B.A. Sci, J.L. Keating and S. Eyres, A Low Back-Specific Version of the SF-36 Physical Functioning Scale, Spine 29 (2004), 586-594.

[8] C.E. Dionne, M. Von Korff, T.D. Koepsell, R.A. Deyo, W.E. Barlow and $\mathrm{H}$. Checkoway, A comparison of pain, functional limitations, and work status indices as outcome measures in back pain research, Spine 24 (1999), 2339-2345. 
[9] J.C. Fanuele, W.A. Abdu, B. Hanscom and J.N. Weinstein, Association between Obesity and Functional Status in Patients with Spine Disease, Spine 27 (2002), 306-312.

[10] K.R. Fontaine, L.J. Cheskin and I. Barofsky, Health-related quality of life in obese persons seeking treatment, J Fam Pract 43 (1996), 265-270.

[11] M. Heliovarra, Body height, obesity, and risk of herniated lumbar intervertebral disc, Spine 12 (1987), 467-472.

[12] Y.S. Horng, Y.H. Hwang, H.C. Wu, H.W. Liang, Y.J. Mhe, F.C. Twu and J.D. Wang, Predicting health-related quality of life in patients with low back pain, Spine 30 (2005), 551-555.

[13] D.A. Hurley, T.E. Dusoir, S.M. McDonough, A.P. Moore, S.J. Linton and G.D. Baxter, Biopsychosocial screening questionnaire for patients with low back pain: preliminary report of utility in physiotherapy practice in Northern Ireland, Clin J Pain 16 (2000), 214-228.
[14] C. Leboeuf-Yde, Body weight and low back pain. A systematic literature review of 56 journal articles reporting on 65 epidemiologic studies, Spine 25 (2000), 226-237.

[15] S.J. Linton and K. Hallden, Can we screen for problematic back pain? A screening questionnaire for predicting outcome in acute and subacute back pain, Clin J Pain 14 (1998), 209 215.

[16] D.L. Patrick, R.A. Deyo, S.J. Atlas, D.E. Singer, A. Chapin and R.B. Keller, Assessing health-related quality of life inpatients with sciatica, Spine 20 (1995), 1899-1908.

[17] A.L. Stewart and R.H. Brook, Effects of being overweight, Am J Public Health 73 (1983), 171-178.

[18] M. van Tulder, A. Malmivaara, R. Esmail and B. Koes, Exercise therapy for low back pain: a systematic review within the framework of the Cochrane collaboration back review group, Spine 25 (2000), 2784-2796. 